\title{
Confluence of Non-Left-Linear TRSs via Relative Termination ${ }^{\star}$
}

\author{
Dominik Klein and Nao Hirokawa \\ School of Information Science \\ Japan Advanced Institute of Science and Technology, Japan \\ \{dominik.klein,hirokawa\}@jaist.ac.jp
}

\begin{abstract}
We present a confluence criterion for term rewrite systems by relaxing termination requirements of Knuth and Bendix' confluence criterion, using joinability of extended critical pairs. Because computation of extended critical pairs requires equational unification, which is undecidable, we give a sufficient condition for testing joinability automatically.
\end{abstract}

\section{Introduction}

Applications in various domains $[16,20,26]$, resulted in an interest in proving confluence of term rewrite systems (TRSs) automatically [3,10,27,28]. Knuth and Bendix [16] showed that confluence of terminating TRSs is decidable by testing joinability of critical pairs, which are induced by overlaps. In the case of non-termination, several powerful techniques have been developed for proving confluence of left-linear systems $[10,23,24]$. Still, proving confluence of both non-left-linear and non-terminating TRSs remains challenging.

Results that tackle this setting can be roughly classified into three categories: First, by generalizing the notion of overlaps, one can formulate direct criteria that guarantee confluence $[8,9]$. The second approach is to decompose a TRS into smaller ones, show confluence of each of them by existing criteria, and formulate modularity conditions to ensure that the union remains confluent $[1,19$, $22]$. The third approach is to generalize Knuth and Bendix' confluence criterion by relaxing termination requirements to relative termination. A famous result here is Jouannaud and Kirchner's criterion for the Church-Rosser modulo property [13] based on extended critical pairs. Geser [6] analyzed their proof to derive confluence criteria based only on syntactical critical pairs.

We present a new confluence criterion that also relies on relative termination, and can be applied for non-left-linear TRSs. The criterion requires to check joinability of extended critical pairs, but we show that under certain conditions, joinability can be concluded from joinability of syntactical critical pairs. With it, we are able to prove confluence of several non-terminating, non-left-linear TRSs fully automatically, for which no known criteria exist.

\footnotetext{
^ This work is supported by the Grant-in-Aids for Young Scientists (B) 22700009 and
} Scientific Research (B) 23300005 of the Japan Society for the Promotion of Science. 
This paper is structured as follows: In Section 2 we recall notions from rewriting, unification, and the decreasing diagram technique, which will be used in proofs later. Our main result is presented in Section 3. Since it requires joinability of uncomputable extended critical pairs, we explain in Section 4 how to automate it, and then report on experiments in Section 5. In Section 6, we compare our criterion with related works, and finally conclude with an outlook on future work in Section 7.

\section{Preliminaries}

We assume familiarity with the basics of term rewriting $([4,21])$.

Term Rewriting. Terms are inductively defined over a set $\mathcal{F}$ of fixed-arity function symbols, and a set $\mathcal{V}$ of variables. For given term $t$, the set of variables occurring in $t$ is denoted by $\mathcal{V} \operatorname{ar}(t)$. The set of (variable, function) positions in $t$ is denoted by $\mathcal{P}$ os $(t)\left(\mathcal{P o s}_{\mathcal{V}}(t), \mathcal{P}_{\mathcal{F}}(t)\right)$. Here positions are expressed by sequences on natural numbers, and the root position $\varepsilon$ is the empty sequence. Given positions $p, q$, and $o$, we write $p \backslash q$ for $o$ if $p=q o$. We write $\triangleright$ for the proper superterm relation. The domain $\operatorname{Dom}(\sigma)$ of a substitution $\sigma$ is the set $\{x \in \mathcal{V} \mid x \neq x \sigma\}$. A rewrite rule $\ell \rightarrow r$ is a pair $(\ell, r)$ of terms with $\mathcal{V} \operatorname{ar}(r) \subseteq \mathcal{V} \operatorname{ar}(\ell)$ and $\ell \notin \mathcal{V}$. A TRS is a collection of rewrite rules. A rewrite rule is left-linear if no variable occurs more than once in $\ell$. Likewise, a TRS is left-linear, if all of its rules are. An extended rewrite rule is a pair $(\ell, r)$ of terms with $\ell \notin \mathcal{V}$, and an extended $T R S(e T R S)$ is a set of extended rewrite rules. A rewrite step of $\mathcal{R}$ at position $p$ is denoted by $\stackrel{p}{\rightarrow}_{\mathcal{R}}$. We write $\downarrow_{\mathcal{R}}$ for the join relation $\rightarrow_{\mathcal{R}}^{*} \cdot{ }_{\mathcal{R}}^{*} \leftarrow$. We write $\rightarrow_{1} / \rightarrow_{2}$ for $\rightarrow_{2}^{*} \cdot \rightarrow_{1} \cdot \rightarrow_{2}^{*}$, and $\rightarrow_{\mathcal{R} / \mathcal{S}}$ for $\rightarrow_{\mathcal{R}} / \rightarrow_{\mathcal{S}}$. $\mathcal{R}$ is relatively terminating over a TRS $\mathcal{S}$ or $\mathcal{R} / \mathcal{S}$ is terminating, if $\rightarrow_{\mathcal{R} / \mathcal{S}}$ is so.

Unification. We briefly recapitulate some notions from unification theory. An equality $s \approx t$ is the ordered pair $(s, t)$ of terms. Let $\mathcal{E}$ and $\mathcal{S}$ be sets of equalities, and $X$ the set of all variables in $\mathcal{E}$. Given a substitution $\sigma$, we write $\mathcal{E} \sigma$ for $\{s \sigma \approx t \sigma \mid s \approx t \in \mathcal{E}\}$. An $\mathcal{S}$-unifier of $\mathcal{E}$ is a substitution $\sigma$ such that $\mathcal{E} \sigma \subseteq \leftrightarrow_{\mathcal{S}}^{*}$. A substitution $\sigma$ is more general than a substitution $\sigma^{\prime}$ on $X\left(\sigma \lesssim_{\mathcal{S}}^{X} \sigma^{\prime}\right)$, if there exists a substitution $\tau$ such that $x \sigma^{\prime} \leftrightarrow_{\mathcal{S}}^{*} x \sigma \tau$ for all $x \in X$. Let $\mathcal{U}$ be a set of $\mathcal{S}$-unifiers of $\mathcal{E}$. We say that $\mathcal{U}$ is complete if for every $\mathcal{S}$-unifier of $\mathcal{E}$ there is a more general element in $\mathcal{U}$. If in addition all elements in $\mathcal{U}$ are minimal with respect to $\lesssim_{\mathcal{S}}^{X}$, we call $\mathcal{U}$ minimal complete. A substitution $\sigma$ is an $\mathcal{S}$-most general unifier $(\mathcal{S}-m g u)$ of $\mathcal{E}$, if $\{\sigma\}$ is a minimal complete set of $\mathcal{S}$-unifiers of $\mathcal{E}$. In the special case of $\mathcal{S}=\varnothing$, we simply speak of (syntactic) unification, unifiers and mgu's. A set of equalities $\mathcal{E}=\left\{x_{1} \approx t_{1}, \ldots, x_{n} \approx t_{n}\right\}$ is in solved form, if $x_{i}$ are pairwise distinct variables, and no $x_{i}$ occurs in $t_{i}$. For $\mathcal{E}$ in solved form, we write $\overrightarrow{\mathcal{E}}$ for the induced substitution $\left\{x_{1} \mapsto t_{1}, \ldots, x_{n} \mapsto t_{n}\right\}$. Note that in general, $\mathcal{S}$-unifiability does not ensure presence of an $\mathcal{S}$-mgu, except for $\mathcal{S}=\varnothing$. 
Critical Pairs. Conditions for confluence are often based on the notion of overlaps and critical pairs. Let $\mathcal{R}_{1}, \mathcal{R}_{2}, \mathcal{S}$ be eTRSs. An $\mathcal{S}$-overlap $\left(\ell_{1} \rightarrow r_{1}, p, \ell_{2} \rightarrow\right.$ $\left.r_{2}\right)_{\sigma}$ of $\mathcal{R}_{1}$ on $\mathcal{R}_{2}$ consists of a variant $\ell_{1} \rightarrow r_{1}$ of a rule in $\mathcal{R}_{1}$ and $\ell_{2} \rightarrow r_{2}$ of a rule in $\mathcal{R}_{2}$, a position $p \in \mathcal{P}_{\mathcal{F}}\left(\ell_{2}\right)$ and a substitution $\sigma$, such that $\left.\ell_{1} \sigma \leftrightarrow{ }_{\mathcal{S}}^{*} \ell_{2}\right|_{p} \sigma$. If $p=\varepsilon$, then $\ell_{1} \rightarrow r_{1}$ and $\ell_{2} \rightarrow r_{2}$ may not be variants of each other. The pair $\left(\ell_{2} \sigma\left[r_{1} \sigma\right]_{p}, r_{2} \sigma\right)$ induced from the overlap is an $\mathcal{S}$-extended critical pair (or simply $\mathcal{S}$-critical pair) of $\ell_{1} \rightarrow r_{1}$ and $\ell_{2} \rightarrow r_{2}$ at $p$, written $\ell_{2} \sigma\left[r_{1} \sigma\right]_{p} \mathcal{R}_{1} \leftarrow \mathcal{S} \infty \rightarrow_{\mathcal{R}_{2}} r_{2} \sigma$. We write $\mathcal{R}_{1} \leftarrow \mathcal{S} \infty \rightarrow_{\mathcal{R}_{2}}$ for $\mathcal{R}_{1} \leftarrow \mathcal{S} \infty \rightarrow_{\mathcal{R}_{2}} \cup \mathcal{R}_{2} \leftarrow \mathcal{S} \infty \rightarrow_{\mathcal{R}_{1}}$. We remark that our definition of $(\mathcal{S}$-)critical pairs includes pairs originating from non-minimal unifiers, which are usually excluded from the definition to guarantee finiteness of critical pairs.

Let $\operatorname{REN}(t)$ denote a linear term resulting from replacing in $t$ each variable occurrence by a fresh variable. We write $\widehat{\mathcal{R}}$ for the eTRS $\{\operatorname{REN}(\ell) \rightarrow r \mid \ell \rightarrow$ $r \in \mathcal{R}\}$. A TRS $\mathcal{S}$ is strongly non-overlapping on $\mathcal{R}$ if $\widehat{\mathcal{S}}$ has no overlaps on $\widehat{\mathcal{R}}$. We write $\operatorname{SNO}(\mathcal{R}, \mathcal{S})$ if both $\mathcal{S}$ is strongly non-overlapping on $\mathcal{R}$, and $\mathcal{R}$ is strongly non-overlapping on $\mathcal{S}$. Left-linear TRSs without critical pairs are called orthogonal. Orthogonal TRSs are confluent. Moreover, Knuth and Bendix' criterion [16] states that $\mathcal{R} \leftarrow \varnothing \varnothing \rightarrow_{\mathcal{R}} \subseteq \downarrow_{\mathcal{R}}$ implies confluence of a terminating TRS $\mathcal{R}$.

Decreasing Diagrams. Van Oostrom showed a powerful confluence criterion for abstract rewrite systems (ARSs), called the decreasing diagram technique [25]. Let $\mathcal{A}=\left(A,\left\langle\rightarrow_{\alpha}\right\rangle_{\alpha \in I}\right)$ be an ARS and $>$ a proper order on $I$. For every $\alpha \in I$ we write $\stackrel{\vee}{\rightarrow}_{\alpha}$ for $\left\{\rightarrow_{\beta} \mid \beta \in I\right.$ and $\left.\beta<\alpha\right\}$, and write $\stackrel{\vee}{\rightarrow}_{\alpha}^{*}$ for $\left(\stackrel{\vee}{\rightarrow}_{\alpha}\right)^{*}$. The union

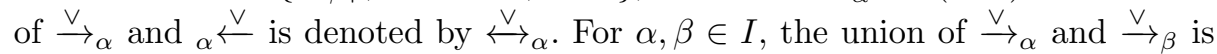

written as $\stackrel{\vee}{\rightarrow}_{\alpha \beta}$. Two labels $\alpha$ and $\beta$ are decreasing with respect to $>$ if

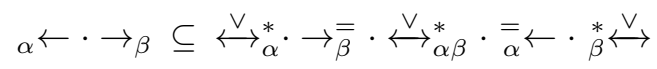

An ARS $\mathcal{A}=\left(A,\left\langle\rightarrow_{\alpha}\right\rangle_{\alpha \in I}\right)$ is decreasing if there exists a well founded order $>$ such that all two labels in $I$ are decreasing with respect to $>$.

Theorem 1 ([25]). A decreasing ARS is confluent.

\section{Confluence Criterion}

First we state our main theorem, which is a proper generalization (when $\mathcal{S} \neq \varnothing$ ) of Knuth and Bendix' confluence criterion.

Theorem 2. Suppose that $\mathcal{S}$ is confluent, $\mathcal{R} / \mathcal{S}$ is terminating, and $\operatorname{SNO}(\mathcal{R}, \mathcal{S})$. The union $\mathcal{R} \cup \mathcal{S}$ of the TRSs is confluent if and only if $\leftarrow_{\mathcal{R}} \leftarrow \mathcal{S}^{\infty} \rightarrow_{\mathcal{R}} \subseteq \downarrow_{\mathcal{R} \cup \mathcal{S}}$.

In the rest of this section we first prove our main theorem, and afterwards give examples of its application. Let $\mathcal{R}$ and $\mathcal{S}$ be TRSs. We introduce an intermediate relation $\rightarrow$, such that $\rightarrow_{\mathcal{R} \cup \mathcal{S}} \subseteq \rightarrow \subseteq \rightarrow_{\mathcal{R} \cup \mathcal{S}}^{*}$. Confluence of this intermediate 
relation readily implies confluence of $\mathcal{R} \cup \mathcal{S}$. The relation $\rightarrow$ is defined as the union of $\rightarrow_{\mathcal{R}_{\mathcal{S}}}$ and $\rightarrow_{\mathcal{S}}^{*}$, where $\mathcal{R}_{\mathcal{S}}$ is the TRS

$$
\left\{\ell^{\prime} \sigma \rightarrow r \tau \mid \ell^{\prime} \rho \rightarrow r \in \mathcal{R} \text { and } \sigma \rightarrow_{\mathcal{S}}^{*} \rho \tau \text { for some substitution } \rho \text { on } \mathcal{V}\right\}
$$

In the above set $\sigma \rightarrow_{\mathcal{S}}^{*} \tau$ means that $x \sigma \rightarrow_{\mathcal{S}}^{*} x \tau$ for all variables $x$. It is important to note that in the definition linearity of $\ell^{\prime}$ can be assumed without loss of generality, and that the inclusions $\rightarrow_{\mathcal{R}} \subseteq \rightarrow_{\mathcal{R}_{\mathcal{S}}} \subseteq \rightarrow_{\mathcal{S}}^{*} \cdot \rightarrow_{\mathcal{R}}$ hold.

We show confluence of $\rightarrow$ by the decreasing diagram technique with the predecessor labeling [25]: We write $b \rightarrow{ }_{a} c$ if $a \rightarrow^{*} b \rightarrow c$. Labels are compared with respect to $\rightarrow_{\mathcal{R} / \mathcal{S}}^{+}$, denoted by $>$. Since termination of $\mathcal{R} / \mathcal{S}$ is presupposed in the theorem, the relation $>$ forms a well-founded order. The next lemma states a property of rewriting in substitutions.

Lemma 3. If $\sigma \stackrel{p}{\rightarrow}_{\mathcal{R}} u$ and $p \notin \operatorname{Pos}_{\mathcal{F}}(t)$ then $u \rightarrow_{\mathcal{R}}^{*}$ t $\tau$ for some $\tau$ with $\sigma \rightarrow \overline{\overline{\mathcal{R}}} \tau$.

Proof. Suppose $t \sigma \stackrel{p}{\rightarrow}_{\mathcal{R}} u$ and $p \notin \mathcal{P}^{\circ}$ s $_{\mathcal{F}}(t)$. Then there exists a variable position $q \in \mathcal{P o s}_{\mathcal{V}}(t)$ with $q \leqslant p$ and $u=(t \sigma)\left[\left.u\right|_{q}\right]_{q}$. Let $Q$ be the set of all variable occurrences of $\left.t\right|_{q}$ in $t$. Since $\left.\left.u\right|_{q^{\prime}} \rightarrow_{\mathcal{R}} u\right|_{q}$ holds for all $q^{\prime} \in Q \backslash\{q\}$, we have $u \rightarrow_{\mathcal{R}}(t \sigma)\left[\left.u\right|_{q}\right]_{q^{\prime} \in Q \backslash\{q\}}$. The latter term is identical to $(t \sigma)\left[\left.u\right|_{q}\right]_{q^{\prime} \in Q}$. We define the substitution $\tau$ as follows:

$$
\tau(x)= \begin{cases}\left.u\right|_{q} & \text { if } x=\left.t\right|_{q} \\ x \sigma & \text { otherwise }\end{cases}
$$

One can verify $\sigma \rightarrow \overline{\overline{\mathcal{R}}} \tau$ and $(t \sigma)\left[\left.u\right|_{q}\right]_{q^{\prime} \in Q}=t \tau$. Hence $u \rightarrow_{\mathcal{R}}^{*} t \tau$.

We analyze peaks of the form $\triangleleft \cdot \rightarrow$. According to the definition of $\rightarrow$, they fall into the three cases: (a) ${ }_{\mathcal{S}}^{*} \leftarrow \cdot \rightarrow_{\mathcal{S}}^{*}$, (b) $\mathcal{R}_{\mathcal{S}} \leftarrow \cdot \rightarrow_{\mathcal{S}}^{*}$, and (c) $\mathcal{R}_{\mathcal{S}} \leftarrow \cdot \rightarrow_{\mathcal{R}_{\mathcal{S}}}$. For case (a) we can apply confluence of $\mathcal{S}$ to show decreasingness of the peak. The remaining cases are more complicated. We start with a localized version of (b). In the next Lemmata 4,6 , and 7 we assume $\operatorname{SNO}(\mathcal{R}, \mathcal{S})$ and confluence of $\mathcal{S}$.

Lemma 4. If $t_{\mathcal{R}_{\mathcal{S}}} \leftarrow s \rightarrow_{\mathcal{S}} u$ then $t \rightarrow_{\mathcal{R} \cup \mathcal{S}}^{*} \cdot{ }_{\mathcal{R}_{\mathcal{S}}}^{*} \leftarrow u$.

Proof. We perform induction on $s$. Suppose $t_{\mathcal{R}_{\mathcal{S}}} \stackrel{p}{\leftarrow} s \stackrel{q}{\rightarrow} s u$. By the definition of $\mathcal{R}_{\mathcal{S}}$ we may assume $\ell_{1} \rho \rightarrow r_{1} \in \mathcal{R}$ for some linear term $\ell_{1}$ and $\rho: \mathcal{V} \rightarrow \mathcal{V}$, $\left.s\right|_{p}=\ell_{1} \sigma,\left.t\right|_{p}=r_{1} \tau$, and $\sigma \rightarrow_{\mathcal{S}}^{*} \rho \tau$, as well as $\ell_{2} \rightarrow r_{2} \in \mathcal{S},\left.s\right|_{q}=\ell_{2} \mu$, and $\left.u\right|_{q}=r_{2} \mu$. Due to $\operatorname{SNO}(\mathcal{R}, \mathcal{S})$, neither $p \backslash q \in \mathcal{P}_{\operatorname{os}_{\mathcal{F}}}\left(\ell_{2}\right)$ nor $q \backslash p \in \mathcal{P}_{\operatorname{os}_{\mathcal{F}}}\left(\ell_{1}\right)$ holds. We distinguish several cases concerning the relation of $p$ and $q$.

- Suppose $p=\varepsilon$. Then there is a variable position $q_{1}$ of $x_{1}$ in $\ell_{1}$ with $q_{1} \leqslant q$. Since $\left.x_{1} \rho \tau{ }_{\mathcal{S}}^{*} \leftarrow x_{1} \sigma \rightarrow_{\mathcal{S}} u\right|_{q_{1}}$ holds, we have $\left.x_{1} \rho \tau \rightarrow_{\mathcal{S}}^{*} v{ }_{\mathcal{S}}^{*} \leftarrow u\right|_{q_{1}}$ for some $v$ by confluence of $\mathcal{S}$. We define the substitutions $\mu_{1}$ and $\nu$ as follows:

$$
\mu_{1}(x)=\left\{\begin{array}{ll}
\left.u\right|_{q_{1}} & \text { if } x=x_{1} \\
x \sigma & \text { otherwise }
\end{array} \quad \nu(x)= \begin{cases}v & \text { if } x=x_{1} \rho \\
x \tau & \text { otherwise }\end{cases}\right.
$$

We have $\tau \rightarrow_{\mathcal{S}}^{*} \nu$, and also $u=\ell_{1} \mu_{1}$ by linearity of $\ell_{1}$. Moreover, $\mu_{1} \rightarrow_{\mathcal{S}}^{*} \rho \nu$ because $x \mu_{1} \rightarrow_{\mathcal{S}}^{*} v=x_{1} \rho \nu=x \rho \nu$ if $x=x_{1}$, and $x \mu=x \sigma \rightarrow_{\mathcal{S}}^{*} x \nu$ otherwise. Therefore, we obtain $t=r_{1} \tau \rightarrow_{\mathcal{S}}^{*} r_{1} \nu_{\mathcal{R}_{\mathcal{S}}} \leftarrow \ell_{1} \mu_{1}=u$. 
- Suppose $q=\varepsilon$. We may presume $\mathcal{V} \operatorname{ar}\left(\ell_{1}\right) \cap \mathcal{V} \operatorname{ar}\left(\ell_{2}\right)=\varnothing$, and thus $\sigma=\mu$ can be assumed. Since $\ell_{2} \sigma \rightarrow_{\mathcal{R}_{\mathcal{S}}} t$ holds, by Lemma 3 we obtain $t \rightarrow_{\mathcal{R}_{\mathcal{S}}}^{*} \ell_{2} \nu$ for some $\nu$ with $\sigma \rightarrow \overline{\overline{\mathcal{R}}}_{\mathcal{S}} \nu$. Thus, $t \rightarrow{ }_{\mathcal{R}_{\mathcal{S}}}^{*} \ell_{2} \nu \rightarrow_{\mathcal{S}} r_{2} \nu_{\mathcal{R}_{\mathcal{S}}} \leftarrow r_{2} \sigma=r_{2} \mu=u$.

- If $p=i p^{\prime}$ and $q=j q^{\prime}$ for some $i, j \in \mathbb{N}$ with $i \neq j$, one can easily verify $t \stackrel{q}{\rightarrow} \mathcal{S}_{\mathcal{S}} \cdot \stackrel{p}{\stackrel{p}{\leftarrow}} u$.

- Otherwise, $p=i p^{\prime}$ and $q=i q^{\prime}$ for some $i \in \mathbb{N}$. Since $\left.\left.t\right|_{i} \mathcal{R}_{\mathcal{S}} \leftarrow s\right|_{i} \rightarrow_{\mathcal{S}}$ $\left.u\right|_{i}$ holds, the induction hypothesis yields $\left.\left.t\right|_{i} \rightarrow_{\mathcal{R} \cup \mathcal{S}}^{*} \cdot \mathcal{R}_{\mathcal{S}}^{*} \leftarrow u\right|_{i}$. Therefore $t \rightarrow{ }_{\mathcal{R} \cup \mathcal{S}}^{*} \cdot \mathcal{R}_{\mathcal{S}}^{*} \leftarrow u$.

In order to handle peaks of shape $\mathcal{R}_{\mathcal{S}} \leftarrow \cdot \rightarrow_{\mathcal{S}}^{*}$ we show an auxiliary lemma for ARSs. In the next lemma $\rightarrow$ stands for $\rightarrow_{1} \cup \rightarrow_{2}$ and $>$ for $\left(\rightarrow_{1} / \rightarrow_{2}\right)^{+}$, and we write $b \rightarrow_{a} c$ if $a \rightarrow^{*} b \rightarrow c$. We will freely use the next two facts: (1) for all $a, b, c$ with $a>b$, we have that $b \stackrel{\vee}{\longleftrightarrow}_{a}^{*} \cdot \rightarrow^{*} c$ implies $b \stackrel{\vee}{\hookrightarrow}_{a}^{*} c$, and (2) $b \rightarrow_{1}^{=} \cdot \stackrel{\vee}{\longrightarrow}_{a}^{*} c$ whenever $a \rightarrow^{*} b \rightarrow_{1}^{*} c$.

Lemma 5. Let ${ }_{1} \leftarrow \cdot \rightarrow_{2} \subseteq \rightarrow^{*} \cdot{ }_{1}^{*} \leftarrow$. If $b{ }_{1} \leftarrow a \rightarrow_{2}^{*} c$ then $b \stackrel{\vee}{\longleftrightarrow}_{a}^{*} \cdot{ }_{1} \leftarrow c$.

Proof. Let $b_{1} \leftarrow a \rightarrow_{2}^{n} c$. We show the claim by induction on $n$. If $n=0$ then trivially the claim holds. Otherwise, $a \rightarrow_{2}^{n-1} d \rightarrow_{2} c$ for some $d$. The induction hypothesis yields $b \stackrel{\vee}{\longleftrightarrow}_{a}^{*} e \overline{\overline{1}} \leftarrow d$ for some $e$. We distinguish two cases.

- If $d=e$ then $b \stackrel{\vee}{\longleftrightarrow}{ }_{a}^{*} e=d \rightarrow_{1} c$. Thus $b \stackrel{\vee}{\longleftarrow}_{a}^{*} c$ by (1).

- Suppose $d \rightarrow_{1} e$. Because we have $e{ }_{1} \leftarrow d \rightarrow_{2} c$, by the assumption $e \rightarrow^{*}$ $f{ }_{1}^{*} \leftarrow c$ for some $f$. Since $a \rightarrow_{2}^{*} d \rightarrow_{1} e \rightarrow^{*} f$ holds, we obtain $e \breve{\vee}_{a}^{*} f$ by (2). Moreover, $c \rightarrow_{1}^{*} f$ implies $c \rightarrow_{1}^{=} . \stackrel{\vee}{\rightarrow}_{a}^{*} f$ by (2). Hence, $b \stackrel{\vee}{\longleftrightarrow}_{a}^{*} \cdot{ }_{1} \leftarrow c$.

Lemma 6. If $t_{\mathcal{R}_{\mathcal{S}}} \leftarrow s \rightarrow_{\mathcal{S}}^{*} u$ then $t \stackrel{\vee}{\triangleright_{S}^{*}} \cdot{ }_{\mathcal{R}_{\mathcal{S}}}^{=} \leftarrow u$.

Proof. By Lemma 4 we have that $t_{\mathcal{R}_{\mathcal{S}}} \leftarrow s \rightarrow_{\mathcal{S}} u$ implies $t \rightarrow_{\mathcal{R} \cup \mathcal{S}}^{*} \cdot \mathcal{R}_{\mathcal{S}}^{*} \leftarrow u$. The claim follows by instantiating Lemma 5 with $\rightarrow_{1}$ as $\rightarrow_{\mathcal{R}_{\mathcal{S}}}$ and $\rightarrow_{2}$ as $\rightarrow_{\mathcal{S}}$.

Lastly, peaks of case (c), of shape $\mathcal{R}_{\mathcal{S}} \leftarrow \cdot \rightarrow_{\mathcal{R}_{\mathcal{S}}}$, are considered.

Lemma 7. If $t_{\mathcal{R}_{\mathcal{S}}} \leftarrow s \rightarrow_{\mathcal{R}_{\mathcal{S}}} u$ then $t \stackrel{\vee}{{ }_{s}^{*}} u$ or $t \rightarrow_{\mathcal{S}}^{*} \cdot \mathcal{R}_{\mathcal{R}} \leftarrow \mathcal{S} \infty \rightarrow_{\mathcal{R}} \cdot{ }_{\mathcal{S}}^{*} \leftarrow u$.

Proof. We perform induction on $s$. Suppose $t_{\mathcal{R}_{\mathcal{S}}} \stackrel{p}{\leftarrow} s \stackrel{q}{\rightarrow} \mathcal{R}_{\mathcal{S}} u$. By the definition of $\mathcal{R}_{\mathcal{S}}$ we can assume $\ell_{1} \rho_{1} \rightarrow r_{1}, \ell_{2} \rho_{2} \rightarrow r_{2} \in \mathcal{R}$ for some linear terms $\ell_{1}, \ell_{2}$ and $\rho_{1}, \rho_{2}: \mathcal{V} \rightarrow \mathcal{V}$, and

$$
\begin{array}{rlrl}
\left.s\right|_{p}=\ell_{1} \sigma_{1} & \left.t\right|_{p}=r_{1} \tau_{1} & \sigma_{1} \rightarrow_{\mathcal{S}}^{*} \rho_{1} \tau_{1} \\
\left.s\right|_{q}=\ell_{2} \sigma_{2} & \left.u\right|_{q}=r_{2} \tau_{2} & & \sigma_{2} \rightarrow_{\mathcal{S}}^{*} \rho_{2} \tau_{2}
\end{array}
$$

Except for symmetric cases, the relation of $p$ and $q$ falls into the next four cases:

- Suppose $q=\varepsilon$, and $p \in \operatorname{Pos}_{\mathcal{F}}\left(\ell_{2}\right)$. We have $\left.\ell_{1} \rho_{1} \tau_{1} \mathcal{S}^{*} \leftarrow s \rightarrow_{\mathcal{S}}^{*} \ell_{2}\right|_{p} \rho_{2} \tau_{2}$. Without loss of generality $\operatorname{Var}\left(\ell_{1} \rho_{1}\right) \cap \mathcal{V} \operatorname{ar}\left(\ell_{2} \rho_{2}\right)=\varnothing$, and thus we may assume $\tau=\tau_{1} \cup \tau_{2}$ is a well-defined substitution. The substitution $\tau$ is an $\mathcal{S}$-unifier of $\ell_{1} \rho_{1}$ and $\left.\ell_{2} \rho_{2}\right|_{p}$. Because $x \sigma_{2} \rightarrow_{\mathcal{S}}^{*} x \rho_{2} \tau$ holds for all $x \in \mathcal{V} \operatorname{ar}\left(\ell_{2}\right)$,

$$
t=\left(\ell_{2} \sigma_{2}\right)\left[r_{1} \tau\right]_{p} \rightarrow_{\mathcal{S}}^{*}\left(\ell_{2} \rho_{2} \tau\right)\left[r_{1} \tau\right]_{p} \mathcal{R} \leftarrow \mathcal{S} \infty \rightarrow_{\mathcal{R}} r_{2} \tau=u
$$




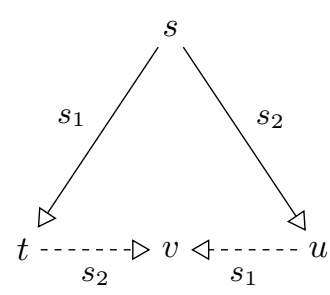

(a) $\stackrel{*}{\mathcal{S}} \leftarrow \cdot \rightarrow_{\mathcal{S}}^{*}$

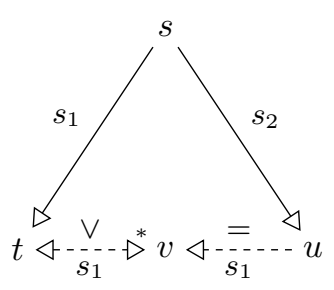

(b) $\mathcal{R}_{\mathcal{S}} \leftarrow \cdot \rightarrow_{\mathcal{S}}^{*}$

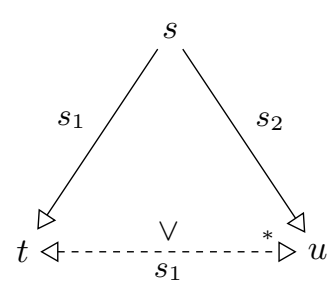

(c) $\mathcal{R}_{\mathcal{S}} \leftarrow \cdot \rightarrow \mathcal{R}_{\mathcal{S}}$

Fig. 1. Decreasingness of $\rightarrow$.

- Suppose $q=\varepsilon$, and $p \notin \mathcal{P o s}_{\mathcal{F}}\left(\ell_{2}\right)$ and $p_{2}$ is a variable occurrence of $x_{2}$ in $\ell_{2}$ with $p_{2} \leqslant p$. Since $\left.t\right|_{p_{2}} \mathcal{R}_{\mathcal{S}} \leftarrow x_{2} \sigma_{2} \rightarrow_{\mathcal{S}}^{*} x_{2} \rho_{2} \tau_{2}$, Lemma 6 yields $\left.t\right|_{p_{2}} \stackrel{\vee}{\longleftrightarrow}{ }_{\left.s\right|_{p_{2}}}^{*}$ $v v_{\mathcal{R}_{\mathcal{S}}}^{=} \leftarrow x_{2} \rho_{2} \tau_{2}$ for some $v$. Because $s=\ell_{2} \sigma_{2},\left.t\right|_{p_{2}} \stackrel{\vee}{\longleftrightarrow}{ }_{\left.s\right|_{p_{2}}}^{*} v$, and $\sigma_{2} \rightarrow_{\mathcal{S}}^{*} \rho_{2} \tau_{2}$ hold, by closure under contexts of rewrite relations and $>$ we obtain

$$
t=\left(\ell_{2} \sigma_{2}\right)\left[\left.t\right|_{p_{2}}\right]_{p_{2}} \stackrel{\vee}{\leftrightarrow}{ }_{s}^{*}\left(\ell_{2} \sigma_{2}\right)[v]_{p_{2}} \rightarrow_{\mathcal{S}}^{*}\left(\ell_{2} \rho_{2} \tau_{2}\right)[v]_{p_{2}}
$$

Thus, $t \stackrel{\vee}{\leftrightarrow}{ }_{s}^{*}\left(\ell_{2} \rho_{2} \tau_{2}\right)[v]_{p_{2}}$. Since $x_{2} \rho_{2} \tau_{2} \rightarrow \overline{\overline{\mathcal{R}}}_{\mathcal{S}} v$ holds and $\ell_{2}$ is linear,

$$
\ell_{2} \rho_{2} \tau_{2} \rightarrow \overline{\overline{\mathcal{R}}}_{\mathcal{S}}\left(\ell_{2} \rho_{2} \tau_{2}\right)[v]_{p_{2}}
$$

is deduced. Here we distinguish two cases. If $\ell_{2} \rho_{2} \tau_{2}=\left(\ell_{2} \rho_{2} \tau_{2}\right)[v]_{p_{2}}$, we obtain

$$
t \stackrel{\vee}{\leftrightarrow}{ }_{s}^{*} \ell_{2} \rho_{2} \tau_{2} \rightarrow_{\mathcal{R}} u
$$

Otherwise, $\ell_{2} \rho_{2} \tau_{2} \rightarrow_{\mathcal{R}_{\mathcal{S}}}\left(\ell_{2} \rho_{2} \tau_{2}\right)[v]_{p_{2}}$. Since by Lemma 3 there exists $\nu$ with $\tau_{2} \rightarrow \overline{\overline{\mathcal{R}}}_{\mathcal{S}} \nu$ such that $\left(\ell_{2} \rho_{2} \tau_{2}\right)[v]_{p_{2}} \rightarrow_{\mathcal{R}_{\mathcal{S}}}^{*} \ell_{2} \rho_{2} \nu$, finally we obtain

$$
t \stackrel{\vee}{\leftrightarrow} *\left(\ell_{2} \rho_{2} \tau_{2}\right)[v]_{p_{2}} \rightarrow_{\mathcal{R}_{\mathcal{S}}}^{*} \ell_{2} \rho_{2} \nu \rightarrow_{\mathcal{R}} r_{2} \nu \mathcal{R}_{\mathcal{S}}^{*} \leftarrow r_{2} \tau_{2}=u
$$

Because $s>t$ and $s>u$ hold, in both cases $t \stackrel{\vee}{{ }_{s}^{*}} u$ is concluded.

- If $p=i p^{\prime}$ and $q=j q^{\prime}$ for some $i, j \in \mathbb{N}$ with $i \neq j$, one can easily verify $t \stackrel{\stackrel{q}{q}_{\rightarrow}}{\mathcal{R}_{\mathcal{S}}} \cdot \mathcal{R}_{\mathcal{S}} \stackrel{p}{\leftarrow} u$, which implies $t \stackrel{\mathfrak{V}^{*}}{\leftrightarrow} u$.

- Otherwise, $p=i p^{\prime}$ and $q=i q^{\prime}$ for some $i \in \mathbb{N}$. Since $\left.\left.\left.t\right|_{i} \mathcal{R}_{\mathcal{S}} \leftarrow s\right|_{i} \rightarrow_{\mathcal{R}_{\mathcal{S}}} u\right|_{i}$ holds, by induction hypothesis $\left.\left.\left.t\right|_{i} \stackrel{\vee}{\longleftrightarrow}_{S}^{*}\right|_{i} u\right|_{i}$ or $\left.\left.t\right|_{i} \rightarrow_{\mathcal{S}}^{*} \cdot \mathcal{R}_{\mathcal{R}} \leftarrow \mathcal{S} \infty \rightarrow_{\mathcal{R}} \cdot \mathcal{S}_{\mathcal{S}}^{*} \leftarrow u\right|_{i}$ is deduced. Thus, $t \stackrel{\vee}{\longleftrightarrow}_{S}^{*} u$ or $t \rightarrow_{\mathcal{S}}^{*} \cdot \mathcal{R}_{\mathcal{R}} \leftarrow \mathcal{S} \infty \rightarrow_{\mathcal{R}} \cdot{ }_{\mathcal{S}}^{*} \leftarrow u$ is concluded.

Now we are ready to prove the main theorem.

Proof (of Theorem 2). Suppose that $\mathcal{S}$ is confluent, $\mathcal{R} / \mathcal{S}$ is terminating, and $\operatorname{SNO}(\mathcal{R}, \mathcal{S})$. We show that $\mathcal{R} \cup \mathcal{S}$ is confluent if and only if $\leftarrow_{\mathcal{R}} \leftarrow \mathcal{S} \infty \rightarrow_{\mathcal{R}} \subseteq \downarrow_{\mathcal{R} \cup \mathcal{S}}$. Since the "only if"-direction is trivial, we only show the "if"-direction. Assume $\mathcal{R} \leftarrow \mathcal{S} \infty \rightarrow_{\mathcal{R}} \subseteq \downarrow_{\mathcal{R} \cup \mathcal{S}}$. Because confluence of $\rightarrow$ implies confluence of $\mathcal{R} \cup \mathcal{S}$, according to Theorem 1 , it is enough to show decreasingness of $\rightarrow$. Let $t_{s_{1}} \leftarrow s \rightarrow s_{2} u$. As mentioned, following the definition of $\rightarrow$, we distinguish three cases. 
(a) If $t \mathcal{S}_{\mathcal{S}}^{*} \leftarrow s \rightarrow_{\mathcal{S}}^{*} u$ then $t \rightarrow_{\mathcal{S}}^{*} v{ }_{\mathcal{S}}^{*} \leftarrow u$ for some $v$ by confluence of $\mathcal{S}$.

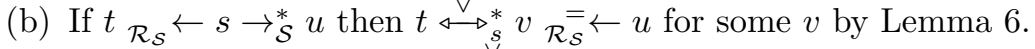

(c) If $t_{\mathcal{R}_{\mathcal{S}}} \leftarrow s \rightarrow_{\mathcal{R}_{\mathcal{S}}} u$ then $t \leftrightarrow \leftrightarrow_{s}^{*} u$ for some $v$ by Lemma 7 and joinability of $\mathcal{S}$-critical pairs.

In all cases decreasingness is established, as seen in Figure 1.

The next examples illustrate Theorem 2. Note that no existing powerful tool can prove their confluence automatically (see Section 5 ).

Example 8. Consider the TRS

$$
\text { 1: } \mathrm{f}(x, x) \rightarrow(x+x)+x \quad 2: x+y \rightarrow y+x
$$

Take $\mathcal{R}=\{1\}$ and $\mathcal{S}=\{2\}$. One can easily verify $\operatorname{SNO}(\mathcal{R}, \mathcal{S})$. Termination of $\mathcal{R} / \mathcal{S}$ can be established using a termination tool such as $\mathrm{T}_{\mathrm{T}} \mathrm{T}_{2} \mathrm{v} 1.06[17]^{1}$, and confluence of $\mathcal{S}$ follows from orthogonality. Because of $\mathcal{R}_{\mathcal{R}} \leftarrow \mathcal{S} \rightarrow_{\mathcal{R}}=\varnothing \subseteq \downarrow_{\mathcal{R} \cup \mathcal{S}}$, we conclude confluence by Theorem 2 .

Example 9. Consider the TRS

$$
\text { 1: } \mathrm{f}(x, x) \rightarrow \mathrm{s}(\mathrm{s}(x)) \quad 2: \infty \rightarrow \mathrm{s}(\infty)
$$

Take $\mathcal{R}=\{1\}$ and $\mathcal{S}=\{2\}$. As in Example 8, one can easily verify the conditions of Theorem 2, including $\mathcal{R} \leftarrow \mathcal{S} \infty \rightarrow_{\mathcal{R}}=\varnothing \subseteq \downarrow_{\mathcal{R} \cup \mathcal{S}}$. Hence the TRS is confluent.

Example 10. Consider the TRS

$$
\begin{aligned}
& \text { 1: eq(s(n), } x: x s, x: y s) \rightarrow \text { eq }(n, x s, y s) \quad 3: \quad \text { nats } \rightarrow 0: \text { inc(nats) } \\
& \text { 2: } \quad \text { eq }(n, x s, x s) \rightarrow \mathrm{T} \quad 4: \operatorname{inc}(x: x s) \rightarrow \mathrm{s}(x): \operatorname{inc}(x s)
\end{aligned}
$$

Take $\mathcal{R}=\{1,2\}$ and $\mathcal{S}=\{3,4\}$. Again, $\operatorname{SNO}(\mathcal{R}, \mathcal{S})$, termination of $\mathcal{R} / \mathcal{S}$ and confluence of $\mathcal{S}$ is established. Moreover, one can show

$$
\mathcal{R} \leftarrow \mathcal{S} \infty \rightarrow_{\mathcal{R}}=\left\{(\mathrm{eq}(s, t, u), \mathrm{T}) \mid s, t, u \text { are terms and } t \leftrightarrow_{\mathcal{S}}^{*} u\right\}
$$

and thus the set is included in $\downarrow_{\mathcal{R} \cup \mathcal{S}}$ because of confluence of $\mathcal{S}$. Hence by using Theorem 2 we conclude that $\mathcal{R} \cup \mathcal{S}$ is confluent.

We conclude this section by mentioning that all conditions of Theorem 2 are essential. One cannot drop $\operatorname{SNO}(\mathcal{R}, \mathcal{S})$ nor termination of $\mathcal{R} / \mathcal{S}$, and even replacing joinability of $\mathcal{S}$-critical pairs by joinability of syntactical critical pairs makes the theorem unsound.

Example 11. Consider Huet's example [11]

$$
\text { 1: } \mathrm{f}(x, x) \rightarrow \mathrm{a} \quad 2: \mathrm{f}(x, \mathrm{~g}(x)) \rightarrow \mathrm{b} \quad 3: \mathrm{c} \rightarrow \mathrm{g}(\mathrm{c})
$$

which is known to be non-confluent. If one takes $\mathcal{R}=\{1\}$ and $\mathcal{S}=\{2,3\}$ then $\mathcal{R} / \mathcal{S}$ is terminating, $\mathcal{S}$ is confluent, and $\mathcal{R} \leftarrow \mathcal{S} \infty \rightarrow_{\mathcal{R}}=\varnothing \subseteq \downarrow_{\mathcal{R} \cup \mathcal{S}}$. If one takes $\mathcal{R}=\{3\}$ and $\mathcal{S}=\{1,2\}$ then, $\operatorname{SNO}(\mathcal{R}, \mathcal{S}), \mathcal{S}$ is confluent, and there are no $\mathcal{S}$-critical pairs of $\mathcal{R}$. Furthermore, if one takes $\mathcal{R}=\{1,2\}$ and $\mathcal{S}=\{3\}$ then $\operatorname{SNO}(\mathcal{R}, \mathcal{S}), \mathcal{R} / \mathcal{S}$ is terminating, $\mathcal{S}$ is confluent, and there are no syntactical critical pairs of $\mathcal{R}$, although $\mathcal{S}$-critical pairs are present.

\footnotetext{
${ }^{1}$ http://colo6-c703.uibk.ac.at/ttt2/
} 


\section{Joinability of $\mathcal{S}$-Critical Pairs}

The biggest challenge in applying Theorem 2 is to check $\mathcal{R}_{\mathcal{S}} \leftarrow_{\mathcal{S}} \rightarrow_{\mathcal{R}} \subseteq \downarrow_{\mathcal{R} \cup \mathcal{S}}$ automatically. The standard approach is to compute a minimal complete set of $\mathcal{S}$-unifiers for $\ell_{1}$ and $\left.\ell_{2}\right|_{p}$ for each combination of rules $\ell_{1} \rightarrow r_{1}, \ell_{2} \rightarrow r_{2}$ and a position $p \in \mathcal{P}_{\mathcal{F}}\left(\ell_{2}\right)$. Then, joinability of its induced critical pairs ensures joinability for all $\mathcal{S}$-unifiers. However, depending on $\mathcal{S}$, the computation of minimal complete sets varies, and worse, minimal complete sets may not even exist for $\mathcal{S}$-unifiable terms. In this section we give sufficient conditions for the joinability and non-joinability of $\mathcal{S}$-critical pairs without performing specific equational unification algorithms.

For the first we show that a most general unifier of strongly $\mathcal{S}$-stable terms is always a most general $\mathcal{S}$-unifier. As the next lemma shows, this allows us to compute $\mathcal{S}$-critical pairs by means of syntactic unification. Here a term $t$ is strongly $\mathcal{S}$-stable if for every position $p \in \mathcal{P}_{\operatorname{os}_{\mathcal{F}}}(t)$ there are no term $u$ and substitution $\sigma$ such that $\left.t\right|_{p} \sigma \rightarrow_{\mathcal{S}}^{*} \cdot \stackrel{\varepsilon}{\rightarrow}_{\mathcal{S}} u$. Note that $t \sigma$ is strongly $\mathcal{S}$-stable if $t$ and $x \sigma$ are strongly $\mathcal{S}$-stable for all variables $x$.

Lemma 12. If $\operatorname{SNO}(\mathcal{R}, \mathcal{S})$ then $\ell$ is strongly $\mathcal{S}$-stable for all $\ell \rightarrow r \in \mathcal{R}$.

In order to show the claim on mgu's, we recall the standard inference rules for syntactic unification from [4]. These rules are defined over sets of equalities on terms.

$\begin{array}{rlr}\text { Eliminate } & \frac{\{x \approx t\} \uplus \mathcal{E}}{\{x \approx t\} \cup \mathcal{E}\{x \mapsto t\}} \quad \text { if } x \notin \mathcal{V} \operatorname{ar}(t) \\ \text { Orient } & \frac{\{t \approx x\} \uplus \mathcal{E}}{\{x \approx t\} \cup \mathcal{E}} \quad \text { if } t \notin \mathcal{V} \\ \text { Delete } & \frac{\{t \approx t\} \uplus \mathcal{E}}{\mathcal{E}} \\ \text { Decompose } & \frac{\left\{f\left(s_{1}, \ldots, s_{n}\right) \approx f\left(t_{1}, \ldots, t_{n}\right)\right\} \uplus \mathcal{E}}{\left\{s_{1} \approx t_{1}, \ldots, s_{n} \approx t_{n}\right\} \cup \mathcal{E}}\end{array}$

We write $\Longrightarrow$ for a derivation by the inferences. The following lemma states that a most general unifier can be computed by a sequence of derivations.

Lemma 13 ([4]). If $s$ and $t$ are unifiable, there exists $\mathcal{E}$ in solved form such that $\{s \approx t\} \Longrightarrow^{*} \mathcal{E}$ and $\overrightarrow{\mathcal{E}}$ is an mgu of $s$ and $t$.

The next lemma shows that the inferences of syntactic unification preserve strong $\mathcal{S}$-stability and $\mathcal{S}$-unifiability. We say that a set $\mathcal{E}$ of equalities is strongly $\mathcal{S}$-stable if $s$ and $t$ are strongly $\mathcal{S}$-stable for all $s \approx t \in \mathcal{E}$.

Lemma 14. Let $\mathcal{S}$ be a confluent TRS. If $\mathcal{E}_{1}$ is strongly $\mathcal{S}$-stable, $\mathcal{E}_{1} \sigma \subseteq \downarrow_{\mathcal{S}}$, and $\mathcal{E}_{1} \Longrightarrow \mathcal{E}_{2}$, then $\mathcal{E}_{2} \sigma \subseteq \downarrow_{\mathcal{S}}$ and $\mathcal{E}_{2}$ is strongly $\mathcal{S}$-stable. 
Proof. Suppose $\mathcal{E}_{1}$ is strongly $\mathcal{S}$-stable, $\mathcal{E}_{1} \sigma \subseteq \downarrow_{\mathcal{S}}$, and $\mathcal{E}_{1} \Longrightarrow \mathcal{E}_{2}$. We distinguish the inference of $\mathcal{E}_{1} \Longrightarrow \mathcal{E}_{2}$. Because the cases of DeLETE and ORIENT are trivial, below we only consider the other two cases:

- Eliminate: Suppose $\mathcal{E}_{1}=\{x \approx t\} \uplus \mathcal{E}^{\prime}$ and $\mathcal{E}_{2}=\{x \approx t\} \cup \mathcal{E}^{\prime} \mu$, where $\mu=\{x \mapsto t\}$ and $x \notin \mathcal{V} \operatorname{ar}(t)$. We claim $\mu \sigma \leftrightarrow_{\mathcal{S}}^{*} \sigma$. Actually it follows from the assumption $x \sigma \downarrow_{\mathcal{S}} t \sigma$. We now prove $\mathcal{E}_{2} \sigma \subseteq \downarrow_{\mathcal{S}}$. It is sufficient to show $u \mu \sigma \downarrow_{\mathcal{S}} v \mu \sigma$ for an arbitrary $u \approx v \in \mathcal{E}^{\prime}$. Because $u \sigma \downarrow_{\mathcal{S}} v \sigma$ by assumption, the claim yields $u \mu \sigma \leftrightarrow_{\mathcal{S}}^{*} v \mu \sigma$. Therefore $u \mu \sigma \downarrow_{\mathcal{S}} v \mu \sigma$ is concluded from confluence of $\mathcal{S}$. To show strong $\mathcal{S}$-stability of $\mathcal{E}_{2}$, fix $u \approx v \in \mathcal{E}^{\prime}$. Since $u, v$, and $x \mu$ are strongly $\mathcal{S}$-stable, so are $u \mu$ and $v \mu$.

- Decompose: Suppose $\mathcal{E}_{1}=\{s \approx t\} \uplus \mathcal{E}^{\prime}$ and $\mathcal{E}_{2}=\left\{s_{1} \approx t_{1}, \ldots, s_{n} \approx\right.$ $\left.t_{n}\right\} \cup \mathcal{E}^{\prime}$ with $s=f\left(s_{1}, \ldots, s_{n}\right)$ and $t=f\left(t_{1}, \ldots, t_{n}\right)$. Since $\mathcal{E}$ is strongly $\mathcal{S}$-stable, and thus $s$ and $t$ are, $s_{i}$ and $t_{i}$ are also strongly $\mathcal{S}$-stable for all $1 \leqslant i \leqslant n$. Furthermore, due to strong $\mathcal{S}$-stability of $s$ and $t, s \sigma \downarrow_{\mathcal{S}} t \sigma$ implies $s_{i} \sigma \downarrow_{\mathcal{S}} t_{i} \sigma$ for all $1 \leqslant i \leqslant n$. Therefore, the claim holds.

We arrive at the aforementioned sufficient condition.

Theorem 15. Let $\mathcal{S}$ be a confluent TRS. An mgu of strongly $\mathcal{S}$-stable terms $s$ and $t$ is an $\mathcal{S}$-mgu of $s$ and $t$.

Proof. Let $\mu$ be an arbitrary mgu of strongly $\mathcal{S}$-stable terms $s$ and $t$. Since $\mu$ is trivially an $\mathcal{S}$-unifier of $s$ and $t$, it is enough to show that $\mu$ is more general than an arbitrary $\mathcal{S}$-unifier $\sigma$ of $s$ and $t$. By using Lemma 13 there is an $\mathcal{E}$ in solved form such that $\{s \approx t\} \Longrightarrow^{*} \mathcal{E}$ and $\overrightarrow{\mathcal{E}}$ is an mgu of $s$ and $t$. Because $s \sigma \leftrightarrow_{\mathcal{S}}^{*} t \sigma$ and $\mathcal{S}$ is confluent, we have $\{s \approx t\} \sigma \subseteq \downarrow_{\mathcal{S}}$, and thus $\mathcal{E} \sigma \subseteq \downarrow_{\mathcal{S}}$ is obtained by induction on the length of $\Longrightarrow$ using Lemma 14. Since $\mathcal{E}$ is in solved form, $x \sigma \downarrow_{\mathcal{S}} x \overrightarrow{\mathcal{E}} \sigma$ holds for all $x \in \operatorname{Dom}(\overrightarrow{\mathcal{E}})$. This means $\sigma \leftrightarrow_{\mathcal{S}}^{*} \overrightarrow{\mathcal{E}} \sigma$. Since $\mu$ is an mgu, there is a substitution $\rho$ with $\overrightarrow{\mathcal{E}}=\mu \rho$. Thus $\sigma \leftrightarrow_{\mathcal{S}}^{*} \mu \rho \sigma$. Hence $\mu$ is more general than $\sigma$.

When automating Theorem 2, confluence of $\mathcal{S}$ and $\operatorname{SNO}(\mathcal{R}, \mathcal{S})$ can be assumed. Therefore, according to Theorem 15 and Lemma 12, a syntactical overlap by an mgu $\mu$ is also an $\mathcal{S}$-overlap by $\mathcal{S}$-mgu $\mu$. Thus joinability of its syntactical critical pairs implies joinability of $\mathcal{S}$-critical pairs induced by any $\mathcal{S}$-unifier.

Example 16 (continued from Example 10). We consider again the example with $\mathcal{R}=\{1,2\}$ and $\mathcal{S}=\{3,4\}$. Take the first and second rules renamed:

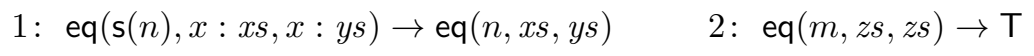

We know that there is an overlap between 1 and 2 at root position with the mgu $\mu=\{m \mapsto \mathrm{s}(n), z s \mapsto x: x s, y s \mapsto x s\}$. Elsewhere, even $\mathcal{S}$-overlaps cannot occur. The induced critical pair (eq $(n, x s, x s), \mathrm{T}$ ) is trivially joinable by the second rule. Hence $\mathcal{R} \leftarrow \mathcal{S} \infty \rightarrow_{\mathcal{R}} \subseteq \downarrow_{\mathcal{R} \cup \mathcal{S}}$ holds.

Confluence of $\mathcal{S}$ cannot be dropped in Theorem 15. 
Example 17. Consider the TRS $\mathcal{S}$

$$
\mathrm{g}(x, y) \rightarrow \mathrm{f}(x, x) \quad \mathrm{g}(x, y) \rightarrow \mathrm{f}(x, y)
$$

The terms $\mathrm{f}\left(x_{1}, x_{1}\right)$ and $\mathrm{f}(x, y)$ are both strongly $\mathcal{S}$-stable, and the substitution $\mu=\left\{x \mapsto x_{1}, y \mapsto x_{1}\right\}$ is a most general unifier. However, $\mu$ is not an $\mathcal{S}$-mgu, because $\mu$ is not more general than the other $\mathcal{S}$-unifier $\left\{x_{1} \mapsto x\right\}$.

Unjoinability of $\mathcal{S}$-critical pairs can be tested similarly to checking nonconfluence of a TRS with the function TCAP ([27]).

Definition 18 ([7]). Let $t$ be a term, and $\mathcal{R}$ a TRS. We define $\operatorname{TCAP}_{\mathcal{R}}(t)$ inductively as a fresh variable, when $t$ is a variable or when $t=f\left(t_{1}, \ldots, t_{n}\right)$ and $\ell$ and $u$ unify for some (renamed) rule $\ell \rightarrow r \in \mathcal{R}$, and $u$, otherwise. Here $u$ stands for $f\left(\operatorname{TCAP}_{\mathcal{R}}\left(t_{1}\right), \ldots, \operatorname{TCAP}_{\mathcal{R}}\left(t_{n}\right)\right)$.

Lemma 19. Let $\ell_{1} \rightarrow r_{1}, \ell_{2} \rightarrow r_{2} \in \mathcal{R}$ and $p \in \mathcal{P o s}_{\mathcal{F}}\left(\ell_{2}\right)$. If $\left.\ell_{1} \sigma \leftrightarrow{ }_{\mathcal{R}}^{*} \ell_{2}\right|_{p} \sigma$, and $\operatorname{TCAP}_{\mathcal{R}}\left(r_{2}\right)$ and $\operatorname{TCAP}_{\mathcal{R}}\left(\ell_{2}\left[r_{1}\right]_{p}\right)$ do not unify, $\mathcal{R}$ is not confluent.

Proof. Using the fact that if $s \sigma \downarrow_{\mathcal{R}} t \tau$ then $\operatorname{TCAP}_{\mathcal{R}}(s)$ and $\operatorname{TCAP}_{\mathcal{R}}(t)$ must unify (see $[27]$ ).

Example 20 (continued from Example 11). Recall $\mathcal{R}=\{1,2\}$ and $\mathcal{S}=\{3\}$ :

$$
\text { 1: } \mathrm{f}(x, x) \rightarrow \mathrm{a} \quad 2: \mathrm{f}(y, \mathrm{~g}(y)) \rightarrow \mathrm{b} \quad 3: \mathrm{c} \rightarrow \mathrm{g}(\mathrm{c})
$$

where variables are renamed in rule 2 . We denote $i$-th rule by $\ell_{i} \rightarrow r_{i}$. While $\ell_{1}$ and $\left.\ell_{2}\right|_{\varepsilon}$ are $(\mathcal{R} \cup \mathcal{S})$-unifiable with $\{x, y \mapsto \mathrm{c}\}, \operatorname{TCAP}_{\mathcal{R} \cup \mathcal{S}}\left(\ell_{2}\left[r_{1}\right]_{\varepsilon}\right)=\mathrm{a}$ and $\operatorname{TCAP}_{\mathcal{R} \cup \mathcal{S}}\left(r_{2}\right)=\mathrm{b}$ do not unify. Thus, by Lemma $19, \mathcal{R} \cup \mathcal{S}$ is not confluent.

In automation we need to test $\mathcal{S}$-unifiability of $\ell_{1}$ and $\left.\ell_{2}\right|_{p}$. This can be automated by first-order theorem provers (for unit equational problems, so-called UEQ) and indeed non-confluence of the above TRS can be proved automatically, see Section 5. Note that in contrast to [27] this approach only requires $\mathcal{S}$-unifiability but not $\mathcal{S}$-unifiers.

As a final remark, from the absence of a unifier we may not conclude nonexistence of $\mathcal{S}$-critical pairs, as illustrated in Example 11.

\section{Experiments}

In order to assess feasibility of our methods, we implemented Theorem 2 together with Theorem 15 for confluence, and Lemma 19 for non-confluence. In the next subsections we mention details of our implementation and report on experimental data. 


\subsection{Implementation}

In order to automate Theorem 2 we employed $\mathrm{T}_{\mathrm{T}} \mathrm{T}_{2}$ v1.06 [17] for checking relative termination $\mathcal{R} / \mathcal{S}$ and an extended version of Maxcomp [14] for testing $\mathcal{S}$-unifiability, using ordered completion. To check confluence of $\mathcal{S}$, we used the existing three state-of-the-art confluence provers: ACP v0.20 $[3]^{2}$, CSI v0.1 $[27]^{3}$, and Saigawa v1.2 [10] $]^{4}$. Since termination of $\mathcal{R} \cup \mathcal{S}$ cannot be assumed, we only test joinability of $\mathcal{S}$-critical pairs by at most four step rewriting for each term.

We give a brief overview of our procedure. Given a TRS $\mathcal{P}$, we output either YES ( $\mathcal{P}$ is confluent), NO ( $\mathcal{P}$ is not confluent), or MAYBE (confluence of $\mathcal{P}$ is neither proven nor disproven). We enumerate all possible partitions $\mathcal{P}=\mathcal{R} \uplus \mathcal{S}$, and then for each $(\mathcal{R}, \mathcal{S})$, we test whether $\operatorname{SNO}(\mathcal{R}, \mathcal{S})$, termination of $\mathcal{R} / \mathcal{S}$, and confluence of $\mathcal{S}$ holds. If one of these conditions does not hold, we continue with the next partition; if none is left, we return MAYBE. Otherwise, to check the last remaining condition of Theorem 2, namely $\mathcal{R} \leftarrow \mathcal{S} \infty \rightarrow_{\mathcal{R}} \subseteq \downarrow_{\mathcal{R} \cup \mathcal{S}}$, we proceed in the following way: For all tuples $\left(\ell_{1} \rightarrow r_{1}, p, \ell_{2} \rightarrow r_{2}\right)$ where $\ell_{1} \rightarrow r_{1}$ and $\ell_{2} \rightarrow r_{2}$ are rules from $\mathcal{R}$ and $p \in \operatorname{Pos}_{\mathcal{F}}\left(\ell_{2}\right)$, we test in the following order:

1. If $\operatorname{REN}\left(\ell_{1}\right)$ and $\operatorname{REN}\left(\left.\ell_{2}\right|_{p}\right)$ are not syntactically unifiable, then no $\mathcal{S}$-overlap exists, and we continue with the next tuple. Otherwise,

2. if $\ell_{1}$ and $\left.\ell_{2}\right|_{p}$ are syntactically unifiable with $\sigma$, the current tuple forms an $\mathcal{S}$-overlap, so we test joinability of the induced critical pair.

(a) If joinability holds, we continue with the next tuple.

(b) If joinability cannot be established, we test whether $\operatorname{TCAP}_{\mathcal{R} \cup \mathcal{S}}\left(r_{2} \sigma\right)$ and $\operatorname{TCAP}_{\mathcal{R} \cup \mathcal{S}}\left(\ell_{2} \sigma\left[r_{1} \sigma\right]_{p}\right)$ syntactically unify. If they are not unifiable, return NO. Otherwise, return MAYBE

3. if $\ell_{1}$ and $\left.\ell_{2}\right|_{p}$ are not syntactically unifiable, we check $\left.\mathcal{S} \models \ell_{1} \approx \ell_{2}\right|_{p}$ by a theorem prover:

(a) If unsatisfiability of the formula is detected, no $\mathcal{S}$-overlap exists, and we continue.

(b) If satisfiability is detected, we test syntactic unifiability of $\operatorname{TCAP}_{\mathcal{R} \cup \mathcal{S}}\left(r_{2}\right)$ and $\operatorname{TCAP}_{\mathcal{R} \cup \mathcal{S}}\left(\ell_{2}\left[r_{1}\right]_{p}\right)$. If they are not unifiable, return No. If they unify, return MAYBE.

(c) Lastly, if the theorem prover does not provide a conclusive answer, return MAYBE

If no tuple remains, we have established ${ }_{\mathcal{R}} \leftarrow \mathcal{S} \infty \rightarrow_{\mathcal{R}} \subseteq \downarrow_{\mathcal{R} \cup \mathcal{S}}$ and return YES. Correctness of the whole procedure can be established using Theorems 2, 15 and Lemmata 12, 19.

\subsection{Experimental Results}

We tested the implementation on a collection of 32 TRSs, consisting of 29 nonleft-linear non-terminating TRSs in the Confluence Problem Database (Cops Nos. $1-116)^{5}$ and Examples 8, 9 and 10. Note that Example 11 is part of the 29

\footnotetext{
${ }^{2}$ http://www.nue.riec.tohoku.ac.jp/tools/acp/

${ }^{3}$ http://cl-informatik.uibk.ac.at/software/csi/

${ }^{4}$ http://www.jaist.ac.jp/project/saigawa/

${ }^{5}$ http://coco.nue.riec.tohoku.ac.jp/
} 
Table 1. Summary of experimental results (32 TRSs)

\begin{tabular}{lrrrrrr}
\hline & ACP & ACP* & CSI & CSI* $^{*}$ & Saigawa & Saigawa* \\
\hline YES & 12 & $\mathbf{1 9}$ & 7 & $\mathbf{1 5}$ & 0 & $\mathbf{1 0}$ \\
NO & 3 & $\mathbf{4}$ & 3 & $\mathbf{3}$ & 0 & $\mathbf{2}$ \\
\hdashline------ & - & - & - & - & - & - \\
MAYBE & 17 & 9 & 17 & 9 & 32 & 20 \\
timeout $(60 \mathrm{sec})$ & 0 & 0 & 5 & 5 & 0 & 0 \\
\hline
\end{tabular}

TRSs. The tests were single-threaded run on a system equipped with an Intel Core Duo L7500 with $1.6 \mathrm{GHz}$ and $2 \mathrm{~GB}$ of RAM using a timeout of 60 seconds.

The results are depicted in Table $1 .{ }^{6}$ Here columns ACP, CSI and Saigawa show results for running the respective tools, and ACP*, CSI* and Saigawa* show results when using the respective tool to show confluence of the $\mathcal{S}$-part in Theorem 2 .

It should be noted, that the criteria implemented by Saigawa apply only to left-linear systems, whereas CSI is able to show confluence of non-left-linear systems by order-sorted decomposition [5], and the implementation of ACP includes criteria based on layer preserving [19] and persistency decompositions [1], and the criterion by Gomi et al. [9].

For overall results, there are twelve TRSs for which confluence can be shown by ACP, CSI and Saigawa combined, in fact however all twelve can be shown by ACP alone. Extending with Theorem 2, there are 19 TRSs, for which confluence can be shown by ACP* ${ }^{*} \mathrm{CSI}^{*}$ or Saigawa* combined. Similar to the standalonecase, ACP* subsumes both other combinations. As for Example 8, 9 and 10, neither CSI, ACP nor Saigawa can show confluence, whereas all CSI*, ACP* and Saigawa* succeed. Out of the nine TRSs that ACP* missed, four TRSs (Cops Nos. 76, 77, 78, 109) contain AC rules, for which most likely the criterion in [13] applies if suitable equational unification algorithms were implemented (see Section 6), and five TRSs (Nos. 16, 24, 26, 27, 47) are variants of Huet's example (Example 11) or Klop's example [15]: $\{\mathrm{f}(x, x) \rightarrow \mathrm{a}, \mathrm{g}(x) \rightarrow \mathrm{f}(x, \mathrm{~g}(x)), \mathrm{c} \rightarrow \mathrm{g}(\mathrm{c})\}$.

\section{Related Work}

Among others, we compare our criterion with three well-known criteria capable of proving confluence of non-left-linear and non-terminating TRSs. Note that for the second criterion below we use reversibility [2] for comparison, because the original criterion requires equational systems for $\mathcal{S}$ rather than rewrite systems. We say that a TRS $\mathcal{S}$ is reversible if $\mathcal{S} \leftarrow \subseteq \rightarrow_{\mathcal{S}}^{*}$.

- Criteria by Non-E-Overlappingness. The criterion by Gomi et al. [8], later extended in [9], is that a root-E-overlapping TRS, that is also strongly

\footnotetext{
${ }^{6}$ Detailed results are available at http://www.jaist.ac.jp/project/saigawa/
} 
weight-preserving or strongly depth-preserving, is confluent. Here E-overlaps are a generalization of overlaps, and strong non-overlappingness plays a major role in deriving sufficient conditions to decide root-E-overlappingness. ${ }^{7}$ A TRS is strongly depth preserving, if for any rewrite rule and any variable appearing in both sides, the minimal depth of the variable occurrences in the left-hand side is greater than or equal to the maximal depth of the right hand side's occurrences. Instead of comparing the depth of the variable directly, one can also assign weights to function symbols and compare the weight of the variable occurrence, where the weight is the sum of the function symbols from root to its occurrence. For details of the definitions we refer to [9]. Consider the following TRS:

$$
\mathrm{f}(x, x) \rightarrow \mathrm{a} \quad \mathrm{c} \rightarrow \mathrm{g}(\mathrm{c}) \quad \mathrm{g}(x) \rightarrow \mathrm{f}(x, x)
$$

Confluence of this TRS can be established, since it is depth-preserving and root-E-overlapping. However Theorem 2 cannot be applied, since the TRS cannot be partitioned into a non-empty $\mathcal{R}$ and $\mathcal{S}$, such that $\mathcal{R} / \mathcal{S}$ is terminating - except for $\mathcal{R}=\varnothing$. On the other hand, weight-preservation and depth-preservation impose strong syntactic restrictions on the variable positions. Consider for example the TRS

$$
\text { 1: } \mathrm{g}(x, x) \rightarrow \mathrm{f}(x) \quad 2: \mathrm{f}(x) \rightarrow \mathrm{f}(\mathrm{f}(x))
$$

By taking $\mathcal{R}=\{1\}$ and $\mathcal{S}=\{2\}$, Theorem 2 can be applied. However the second rule violates both strong depth and strong weight-preservation.

- Criteria by Extended Critical Pairs. In [13], based on the preliminary work in [12], Jouannaud and Kirchner show that the union of a TRS $\mathcal{R}$ and a reversible TRS $\mathcal{S}$ is confluent if $\mathcal{R} / \mathcal{S}$ and $\triangleright / \leftrightarrow_{\mathcal{S}}$ are terminating and

$$
\mathcal{R} \leftarrow \mathcal{S} \infty \rightarrow_{\mathcal{R} \cup \mathcal{S} \cup \mathcal{S}^{-1}} \subseteq \rightarrow_{\mathcal{R}, \mathcal{S}}^{*} \cdot \leftrightarrow_{\mathcal{S}}^{*} \cdot \mathcal{R}, \mathcal{S}^{*} \leftarrow
$$

Here $s \rightarrow_{\mathcal{R}, \mathcal{S}} t$ if there exist a rule $\ell \rightarrow r \in \mathcal{R}$, a position $p \in \mathcal{P}$ os $(s)$, and a substitution $\sigma$, such that $\left.s\right|_{p} \leftrightarrow_{\mathcal{S}}^{*} \ell \sigma$ and $t=s[r \sigma]_{p}$. Note that $\mathcal{S}$ has a serious restriction: The two termination requirements prohibit application when $\mathcal{S}$ is erasing or collapsing, or even when $C[t] \leftrightarrow_{\mathcal{S}}^{*} t$. For instance, Examples 9 and 10 cannot be handled due to this restriction. On the other hand it is applicable for mutually overlapping TRSs $\mathcal{R}$ and $\mathcal{S}$, for example:

$$
\text { 1: } x+x \rightarrow x \quad 2: x+y \rightarrow y+x \quad 3:(x+y)+z \rightarrow x+(y+z)
$$

By taking $\mathcal{R}=\{1\}$ and $\mathcal{S}=\{2,3\}$, one can easily show confluence of $\mathcal{R} \cup \mathcal{S}$ by using their criterion. However, Theorem 2 cannot be applied because $\mathcal{R}$ and $\mathcal{S}$ overlap on each other. This criterion forms a foundation of AC-completion.

\footnotetext{
$7 \mathcal{S}$-overlaps are sometime called $\mathcal{E}$-overlaps but should not be confused with the Eoverlaps defined by Gomi et al. [8], originally introduced by Ogawa [18].
} 
- Criteria by Relative Termination. Geser [6] introduced several pioneering applications of relative termination. A result of particular interest in this context is the following confluence criterion: A TRS $\mathcal{R} \cup \mathcal{S}$ is confluent if $\mathcal{R}$ is left-linear, $\mathcal{S}$ is confluent, and the following two inclusions hold:

$$
\mathcal{S} \leftarrow \varnothing \varnothing \rightarrow_{\mathcal{R}} \subseteq\left(\rightarrow_{\mathcal{S}}^{*} \cdot \mathcal{R} \cup \mathcal{S}^{*} \leftarrow\right) \cup\left(\rightarrow_{\mathcal{R}} \cdot \downarrow_{\mathcal{R} \cup \mathcal{S}}\right) \quad \mathcal{R} \leftarrow \varnothing \varnothing \rightarrow_{\mathcal{R}} \subseteq \downarrow_{\mathcal{R} \cup \mathcal{S}}
$$

In contrast to Theorem 2, overlaps between rules in $\mathcal{R}$ and $\mathcal{S}$ pose no problem. The following example, due to Geser, shows the power of his approach beyond pure left-linear systems:

$$
\text { 1: } \mathrm{c}(\mathrm{s}(x), \mathrm{s}(y)) \rightarrow \mathrm{c}(x, y) \quad 2: \mathrm{c}(x, x) \rightarrow \mathrm{f}(\mathrm{c}(x, x))
$$

Then confluence can be established by taking $\mathcal{R}=\{1\}$ and $\mathcal{S}=\{2\}$, whereas Theorem 2 is not applicable. ${ }^{8}$ The reason for being able to handle overlaps between $\mathcal{R}$ and $\mathcal{S}$ is, that with the restriction of left-linearity of the $\mathcal{R}$ part, joinability of syntactical critical pairs suffices to establish confluence. On the other hand, the requirement of left-linearity prevents application for Examples 8, 9 and 10, except for choosing $\mathcal{R}=\varnothing$.

\section{Conclusion}

In this paper we showed a generalization of Knuth and Bendix' confluence criterion, which can deal with non-left-linear, non-terminating TRSs. Moreover we presented its automation technique. As seen in Section 6, conditions required in our criterion are related to the results by Jouannaud and Kirchner [13] and Geser [6]. Any of them exploits relative termination to overcome non-termination, however still relative termination poses a strict restriction. We anticipate that use of critical pair steps [10] relaxes this restriction.

Acknowledgements. We thank the anonymous referees for their valuable comments.

\section{References}

1. Aoto, T., Toyama, Y.: Persistency of confluence. Journal of Universal Computer Science 3(11), 1134-1147 (1997)

2. Aoto, T., Toyama, Y.: A Reduction-Preserving Completion for Proving Confluence of Non-Terminating Term Rewriting Systems. In: Proc. 22nd RTA. LIPIcs, vol. 10, pp. 91-106 (2011)

3. Aoto, T., Yoshida, J., Toyama, Y.: Proving confluence of term rewriting systems automatically. In: Proc. 21st RTA. LNCS. pp. 93-102 (2009)

4. Baader, F., Nipkow, T.: Term rewriting and all that. Cambridge University Press (1998)

5. Felgenhauer, B., Zankl, H., Middeldorp, A.: Layer systems for proving confluence. In: Proc. 31st FSTTCS. LIPIcs, vol. 13, pp. 288-299 (2011)

${ }_{8}$ All current confluence tools fail to show confluence of the one rule TRS of rule 2 . 
6. Geser, A.: Relative Termination. PhD thesis, Universität Passau (1990). Available as technical report 91-03.

7. Giesl, J., Thiemann, R., Schneider-Kamp, P.: Proving and disproving termination of higher-order functions. In: Proc. 5th FroCoS. LNAI, vol. 3717, pp. 216-231 (2005)

8. Gomi, H., Oyamaguchi, M., Ohta, Y.: On the Church-Rosser property of non-Eoverlapping and strongly depth-preserving term rewriting systems. Trans. IPSJ 37(12), 2147-2160 (1996)

9. Gomi, H., Oyamaguchi, M., Ohta, Y.: On the Church-Rosser property of root-Eoverlapping and strongly depth-preserving term rewriting systems. Trans. IPSJ 39(4), 992-1005 (1998)

10. Hirokawa, N., Middeldorp, A.: Decreasing diagrams and relative termination. Journal of Automated Reasoning 47, 481-501 (2011)

11. Huet, G.: Confluent reductions: Abstract properties and applications to term rewriting systems: Abstract properties and applications to term rewriting systems. Journal of the ACM 27, 797-821 (1980)

12. Jouannaud, J.P.: Confluent and coherent equational term rewriting systems: Application to proofs in abstract data types. In: Proc. 8th CAAP. LNCS, vol. 159, pp. 269-283 (1983)

13. Jouannaud, J.P., Kirchner, H.: Completion of a set of rules modulo a set of equations. SIAM Journal on Computing 15(4), 1155-1194 (1986)

14. Klein, D., Hirokawa, N.: Maximal completion. In: Proc. 22nd RTA. LIPIcs, vol. 10, pp. 71-80 (2011)

15. Klop, J.: Combinatory reduction systems. PhD thesis, Utrecht University (1980)

16. Knuth, D., Bendix, P.: Simple word problems in universal algebras. In: Computational Problems in Abstract Algebra. 263-297 (1970)

17. Korp, M., Sternagel, C., Zankl, H., Middeldorp, A.: Tyrolean Termination Tool 2. In: Treinen, R. (ed.) Proc. 20th RTA 2009. LNCS, vol. 5595, pp. 295-304 (2009)

18. Ogawa, M.: Chew's theorem revisited -uniquely normalizing property of nonlinear term rewriting systems-. In: ISAAC. LNCS, vol. 650, pp. 309-318 (1992)

19. Ohlebusch, E.: Modular properties of composable term rewriting systems. Journal of Symbolic Computation 20, 1-41 (1995)

20. Stump, A., Kimmell, G., Omar, R.E.H.: Type preservation as a confluence problem. In: Proc. 22nd RTA. LIPIcs, vol. 10, pp. 345-360 (2011)

21. TeReSe: Term Rewriting Systems. vol. 55 of Cambridge Tracts in Theoretical Computer Science. Cambridge University Press (2003)

22. Toyama, Y.: On the Church-Rosser property for the direct sum of term rewriting systems. Journal of the ACM 34(1), 128-143 (1987)

23. Toyama, Y.: Commutativity of term rewriting systems. In: Programming of Future Generation Computers II. North-Holland 393-407 (1988)

24. van Oostrom, V.: Developing developments. Theoretical Computer Science 175(1), 159-181 (1997)

25. van Oostrom, V.: Confluence by decreasing diagrams. In: Proc. 19th RTA. LNCS, vol. 5117, pp. 306-320 (2008)

26. Yamamoto, A.: Completeness of extending unification based on basic narrowing. In: Proc. 7th Conference on Logic Programming. LNCS, vol. 383, pp. 1-10 (1988)

27. Zankl, H., Felgenhauer, B., Middeldorp, A.: CSI - a confluence tool. In: Proc. 23th CADE. LNAI, vol. 6803, pp. 499-505 (2011)

28. Zankl, H., Felgenhauer, B., Middeldorp, A.: Labelings for decreasing diagrams. In: Proc. 22nd RTA. LIPIcs. pp. 377-392 (2011) 